\title{
Músculos Papilares en el Corazón del Avestruz (Struthio camelus)
}

\author{
Papillary Muscles in the Heart of the Ostrich (Struthio camelus)
}

\author{
"Marco Figueroa \& ** Jorge Henríquez-Pino
}

FIGUEROA, M. \& HENRÍQUEZ-PINO, J. Músculos papilares en el corazón del avestruz (Struthio camelus). Int. J. Morphol., 27(2):435-440, 2009.

RESUMEN: El corazón del avestruz (Struthio camelus), al igual que el corazón de las aves domésticas, tiene una valva atrioventricular derecha muscular sin cuerdas tendinosas, y una valva atrioventricular izquierda membranosa con cuerdas tendinosas que se extienden entre las cúspides y 3 músculos papilares. El objetivo del estudio fue describir los músculos papilares, número, ubicación y algunas características morfológicas. Se utilizaron 50 corazones de avestruz (Struthio camelus), que fueron disecados y fijados en formalina tamponada al 10\%, y se obtuvieron cortes histológicos de 7 micras los cuales fueron teñidos con Van Gieson y H-E. El estudio macroscópico mostró ausencia de músculos papilares en el ventrículo derecho y en el ventrículo izquierdo 3 masas musculares formadas por trabéculas carnosas longitudinales que dan inserción a las cuerdas tendinosas. En los cortes histológicos se observó que los puntos de inserción dorsal y ventral de la valva atrioventricular derecha a la pared ventricular, son prolongaciones del músculo que forma la valva y presentan fibras conductoras cardíacas. En el ventrículo izquierdo, las trabéculas carnosas longitudinales también presentan fibras conductoras cardíacas las que se extienden hasta la zona de inserción de las cuerdas tendinosas. Los resultados muestran que el corazón del avestruz (Struthio camelus) no tiene músculos papilares de forma cónica como en el corazón humano, son formaciones musculares más desarrolladas que en el corazón de las aves domésticas y funcionalmente actúan como músculos papilares al presentar fibras conductoras cardíacas.

PALABRAS CLAVE: Anatomía; Corazón; Avestruz; Músculos papilares.

\section{INTRODUCCIÓN}

El avestruz pertenece a un grupo de aves denominadas ratites, cuyas especies más importantes desde el punto de vista comercial son el avestruz originario de Africa, el emú de Australia y el ñandú de Sudamérica. Ratites es un término inglés que comprende un grupo de aves que se caracterizan por ser buenas corredoras, que han perdido su capacidad de volar (por lo que carecen de músculos pectorales y de quilla en el esternón) y que anidan en el suelo. El avestruz se agrupa de acuerdo a la siguiente clasificación taxonómica: Clase Aves, Orden Estrucioniformes, Familia Estrucionidae, Género Struthio y Especie Camelus.

La variedad comercial que se reproduce en Chile corresponde a un híbrido, el Struthio camelus var. domesticus, denominado African Black, que se originó principalmente en las subespecies sudafricanas S.c. australis, S.c. camelus y S.c. syriacus, entre otras. La explotación del avestruz se

* Universidad del Bio-Bio, Chillán, Chile.

** Facultad de Medicina, Universidad de La Frontera, Temuco, Chile

Tesis presentada a la Universidad de La Frontera, para la obtención del grado académico de Magíster en Ciencias con mención en Morfología.

Patrocinado por la Universidad del Bío-Bío, Chillán, Chile. presenta como una nueva alternativa para diversificar la agricultura en nuestro país. La cría del avestruz se ha convertido en una industria de rápido crecimiento, que ha pasado a ser una fuente interesante de ingresos. Se reproducen hasta los 40 años, y producen anualmente cinco veces más volumen de carne y cuero que lo que produce el ganado vacuno. Además, ocupan poco espacio y tienen una mejor conversión alimenticia. Los productos que se pueden obtener de esta ave son carne, piel, plumas, aceite, y huevos. La carne de avestruz tiene buen sabor y es similar en textura y apariencia a la carne de ganado vacuno. Sumamente alta en hierro y proteínas, es considerablemente más baja en grasa, colesterol y calorías que la de vacuno.

Los polluelos recién nacidos miden cerca de $25 \mathrm{~cm}$ de altura y pesan aproximadamente $1 \mathrm{Kg}$. En promedio, el polluelo crece $25 \mathrm{~cm}$ por mes durante los seis primeros me- 
ses. De los 10 a los 14 meses de edad están listos para el matadero, pesan normalmente alrededor de $110 \mathrm{~kg}$ y rinden aproximadamente un $60 \%$ luego de faenados. El avestruz produce también 1 a $2 \mathrm{~kg}$ de plumas usables (Ministerio de Agricultura de Chile, 1996).

Macalister (1864) describió el corazón del avestruz (Struthio camelus) con una valva atrioventricular derecha muscular unida a la parte ventral de la pared del ventrículo derecho por una banda muscular, y una valva atrioventricular izquierda con tres cúspides membranosas unidas por cuerdas tendinosas sin músculos papilares conectados a ellas. Estudios posteriores señalan que el corazón del avestruz al igual que el corazón de las aves domésticas, tiene una valva atrioventricular derecha muscular sin cuerdas tendinosas, y una valva atrioventricular izquierda tricúspide con cuerdas tendinosas unidas a tres músculos papilares, dos se encuentran en la pared lateral del ventrículo izquierdo y el tercero en el septum interventricular (Shaner, 1923; Adams, 1937; Bezuidenhout, 1981, 1983; Baumel, 1993: Sisson, 2002; Brólio et al., 2003; König, 2009; Tadjalli et al., 2009).

El objetivo de este trabajo fue describir los músculos papilares, señalar el número y ubicación de éstos en cada ventrículo, realizar un estudio mesoscópico y determinar algunas características morfológicas a través de un estudio histológico.

\section{MATERIAL Y MÉTODO}

Para el estudio macroscópico se utilizaron 50 corazones de avestruz (Fig. 1), y 10 corazones de avestruz para el estudio mesoscópico, de la clase African Black, cuyas edades variaban entre los 12 y 15 meses. Las aves estaban sanas con un peso que oscilaba entre los 72 y $122 \mathrm{~kg}$ (En promedio 92 $\mathrm{Kg}$ ). Los corazones tienen un peso promedio de $678 \mathrm{~g}$ con un máximo de 953 g y un mínimo de 456 g, con una desviación standard de 135,9. Estas aves provienían del criadero "El Carmen" de Calera de Tango, Región Metropolitana, Chile.

Los corazones fueron retirados e identificados mediante el uso de placas plásticas numeradas y sometidos a lavados con agua corriente, se desangraron mediante masajes por 2 horas y luego se fijaron en formalina al 10\% durante 10 días. Se hizo disección macroscópica con el propósito de retirar los atrios, el epicardio y la grasa y se realizó una incisión con tijeras desde el anillo atrioventricular entre las cúspides anterior y posterior, en dirección al ápice del corazón siguiendo el margen de éste, para dejar visibles los músculos papilares en cada ventrículo. Posteriormente, 10 muestras fueron sometidas a una disección mesoscópica de los

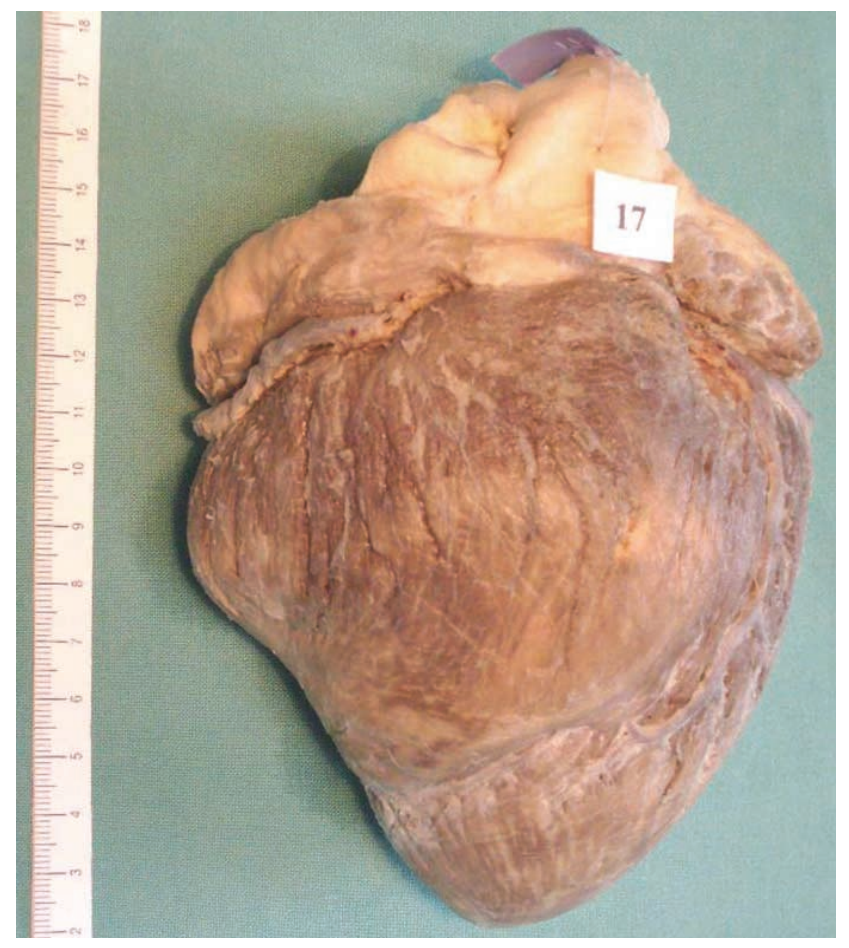

Fig. 1. Corazón de avestruz (Struthio Camelus).

músculos papilares para lo cual se usó una Lupa estereoscópica Olimpus con aumentos de 10 a 50 x, pinzas y tijeras. Para la medición de la longitud de los ventrículos y los músculos papilares, se utilizó un caliper digital. Los ventrículos se midieron desde la base hasta el ápice; para la ubicación de los músculos papilares en el ventrículo izquierdo se midió la longitud entre el ápice del corazón y el punto de inserción de las cuerdas tendinosas. En el ventrículo derecho se midió la longitud entre el ápice y los puntos de inserción dorsal y ventral de la valva atrioventricular derecha en la pared ventricular. También se hicieron cortes histológicos de $7 \mu \mathrm{m}$ con tinción Hematoxilina-Eosina y Van Gieson para determinar la distribución de las fibras musculares que los constituyen.

Para el análisis estadístico de los resultados se usó el programa SPSS 15.0.

\section{RESULTADOS}

La valva atrioventricular derecha del corazón del avestruz (Struthio camelus), es una banda muscular de forma rectangular dispuesta en sentido dorso-ventral que se inserta por sus extremos a la pared lateral y medial del ventrículo derecho. Se observan fibras de conducción cardíaca en las inserciones dorsal y ventral de la valva a la pared ventricular (Fig. 2, 3, 4, 5, 6). 

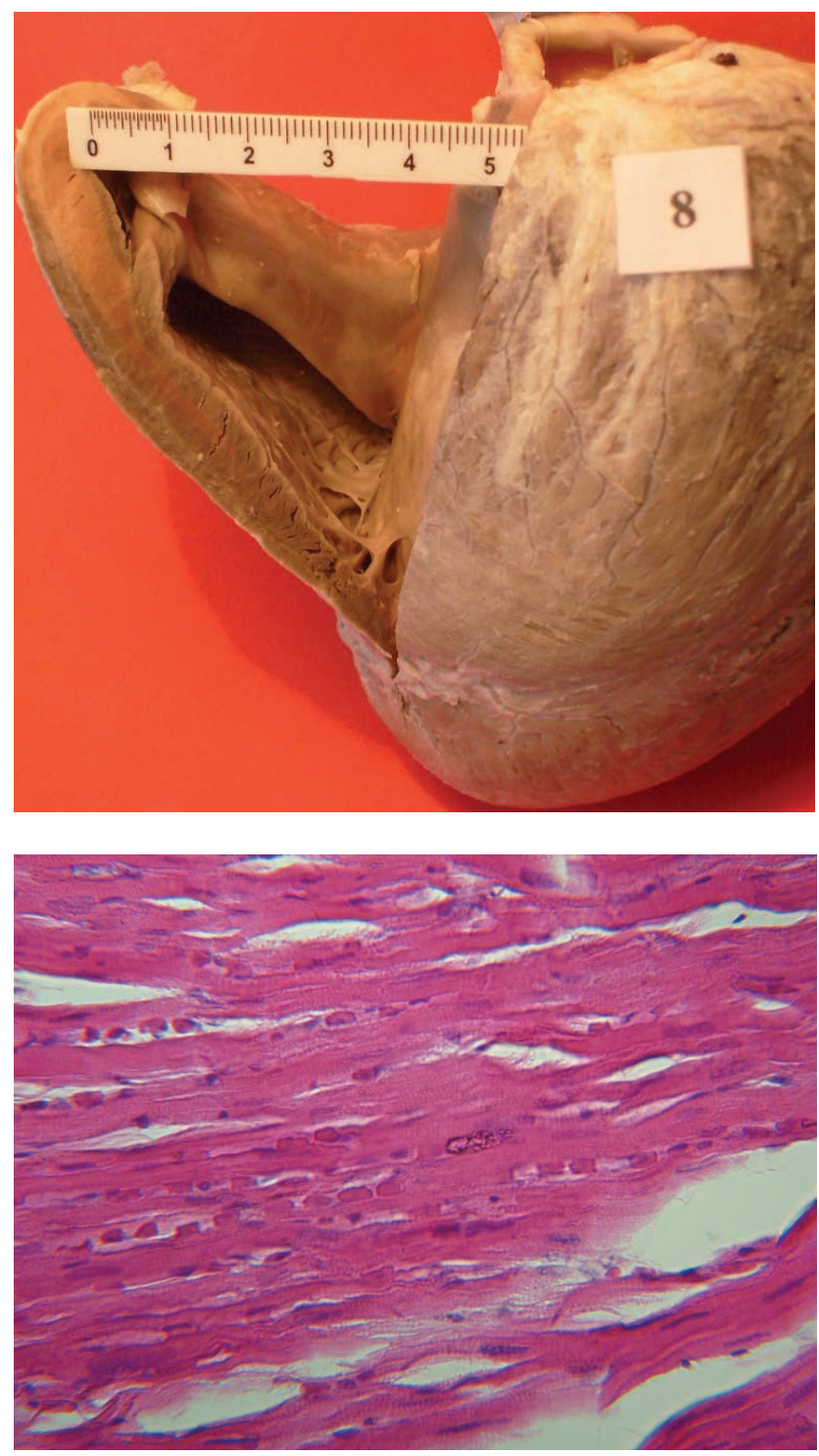

Fig. 4. Microfotografía del corte longitudinal de la Fig. 3 que muestra gran cantidad de fibras de conducción cardíaca (FP) . H.E. 400x.

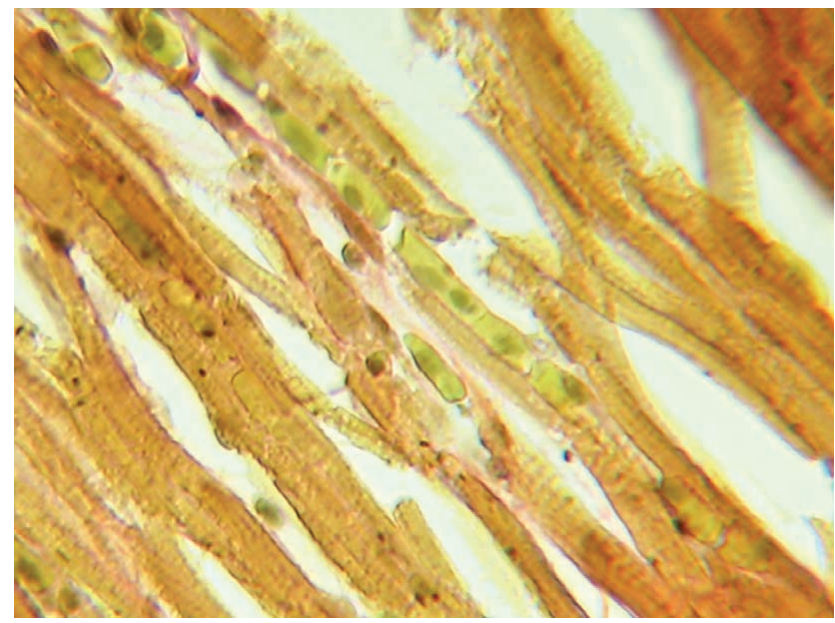

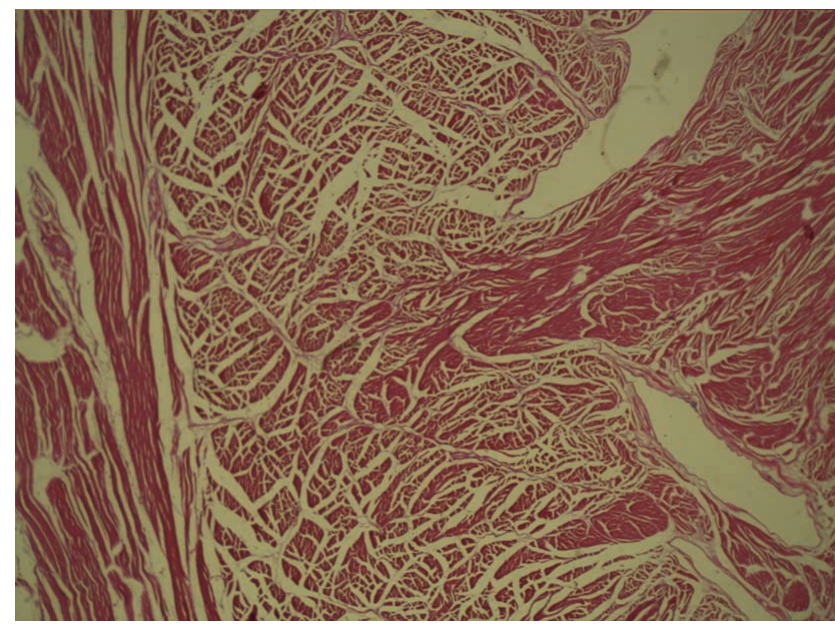

Fig. 2. Valva atrioventricular derecha y sus puntos de inserción dorsal (D) y ventral (V).

Fig. 3. Corte longitudinal de la inserción ventral de la valva atrioventricular derecha (VAVD) a la pared ventricular (VD), donde se observan las fibras musculares que salen desde la valva. H.E. 12,5x.

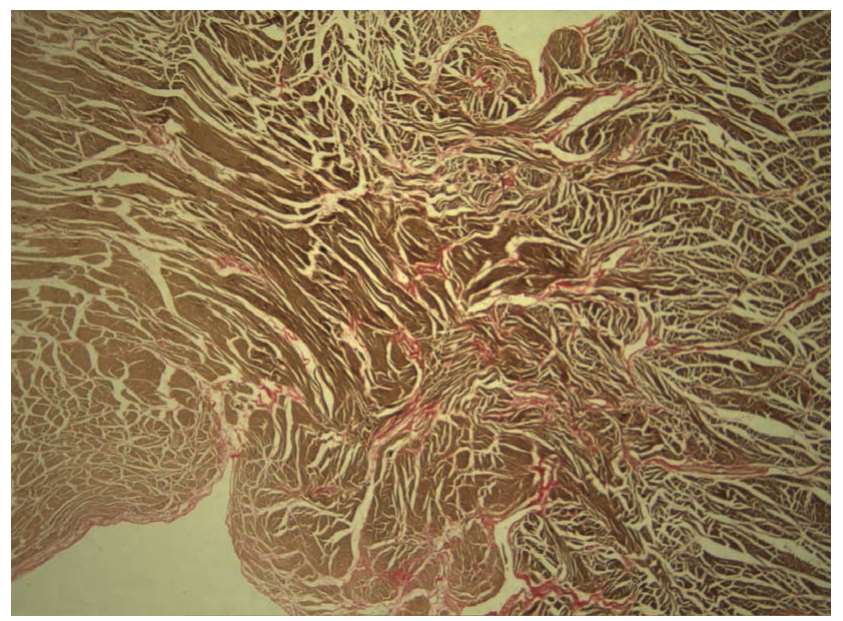

Fig. 5. Corte longitudinal de la inserción dorsal de la valva atrioventricular derecha (VAVD) a la pared ventricular (VD). Van Gieson. 8x.

La longitud ventricular entre la base y el ápice en promedio es de 119,3 $\mathrm{mm}$ con un máximo de 144,2 $\mathrm{mm}$ y un mínimo de 98,5mm, con una desviación standard de 10,2. La inserción ventral promedio de la valva atrioventricular derecha está a 103,4 mm del ápice (de caudal hacia craneal), con un máximo de $123,1 \mathrm{~mm}$ y un mínimo de $94,3 \mathrm{~mm}$, con una desviación standard de 7,9. La inserción dorsal promedio está a 63,5 mm del ápice con un máximo de 74,3 mm y un mínimo de 52,4 mm, con una desviación standard de 5,5. Esta medición demuestra que la inserción ventral de la valva atrioventricular derecha se encuentra en una posición más craneal que la inserción dorsal (Tabla I).

Fig. 6. Microfotografía del corte longitudinal de la Fig. 5 que muestra fibras de conducción cardíaca en color verde. Van Gieson. 400x. 
Sólo en un corazón se encontró una tercera inserción a la pared lateral del ventrículo derecho que se origina de la parte media de la valva atrioventricular derecha, semejante a una trabécula septomarginal (Fig. 7).

En el ventrículo izquierdo, las cuerdas tendinosas se insertan en tres formaciones musculares descritas como músculos papilares anterior, posterior y septal. Estos músculos papilares se forman por la convergencia de largas trabéculas carnosas que provienen desde el ápice hacia la parte craneal del ventrículo izquierdo y no tienen una forma definida pues se presentan como una masa muscular abultada (Fig. 8). En los cortes histológicos se encontraron fibras de conducción cardíaca, a lo largo de las trabéculas y que alcanzan a los músculos papilares (Fig. 9, 10). El número de trabéculas que forman cada M. papilar se detalla en la Tabla II.

En cuanto a la posición de los músculos papilares en la pared ventricular, el músculo papilar anterior se encuentra en promedio a $72,5 \mathrm{~mm}$. del ápice (de caudal hacia craneal), con una desviación standard de 6,3; el M. papilar septal está a 73,5 mm. del ápice, con una desviación standard de 6,5; y el M. papilar posterior a 71,2 $\mathrm{mm}$, con una desviación standard de 6,1 .
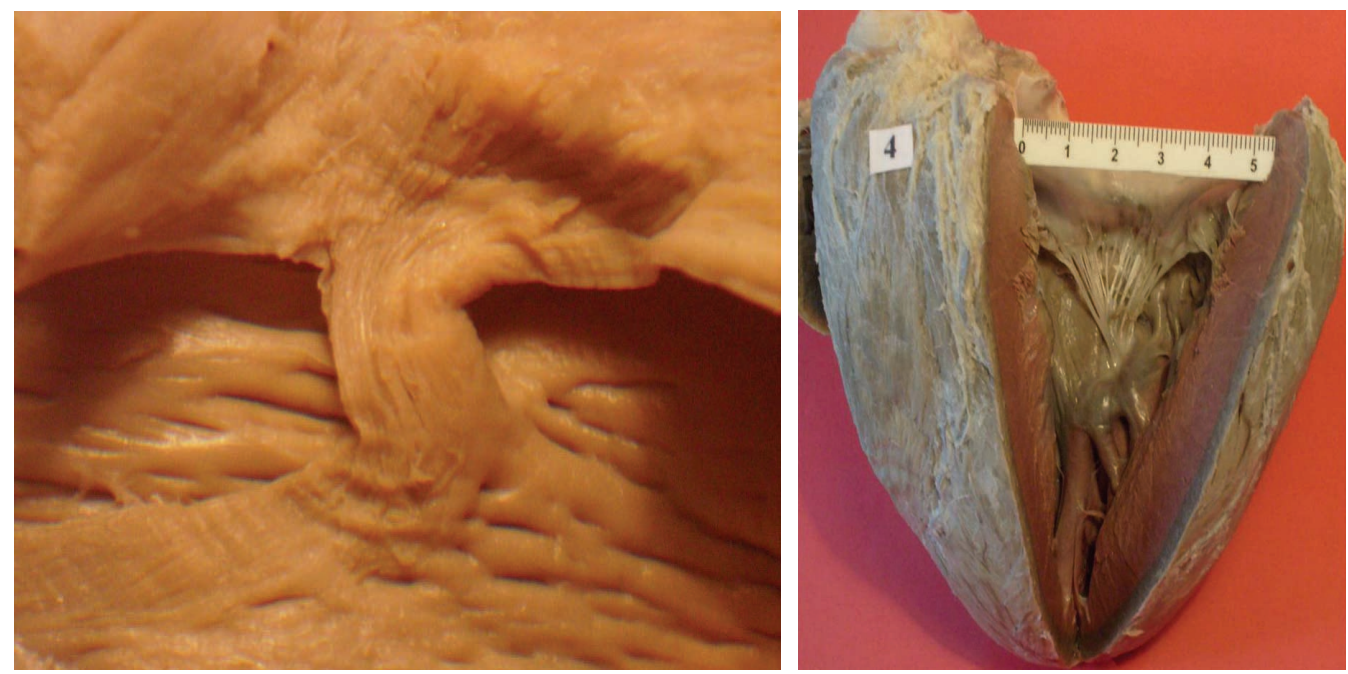

Fig. 8. Músculo papilar posterior del ventrículo izquierdo del corazón de avestruz (Struthio camelus) mostrando la zona de donde se obtuvo el corte histológico.

Fig. 7. Variación de una tercera inserción de la valva atrioventricular derecha a la pared ventricular del corazón de avestruz (Struthio camelus).

Tabla I. prueba de T de Student que señala diferencia significativa entre los puntos de inserción de la valva atrioventricular derecha.

\begin{tabular}{|c|c|c|c|c|c|c|c|c|c|}
\hline \multirow[b]{4}{*}{ Par 1} & & \multicolumn{5}{|c|}{ Diferencias relacionadas } & & \multirow[b]{3}{*}{ gl } & \multirow[b]{3}{*}{$\begin{array}{c}\text { Sig. } \\
\text { (bilateral }\end{array}$} \\
\hline & & & & & $\begin{array}{l}95 \% \text { inter } \\
\text { para }\end{array}$ & $\begin{array}{l}\text { confianzi } \\
\text { encia }\end{array}$ & & & \\
\hline & & Media & D. S. & $\begin{array}{l}\text { Error tip. } \\
\text { de la media }\end{array}$ & inferior & superior & $\mathrm{t}$ & & \\
\hline & $\begin{array}{l}\text { Inserción V- } \\
\text { VAVD } \\
\text { Inserción D- } \\
\text { VAVD }\end{array}$ & 39,9 & 6,0 & .85 & 38,2 & 41,6 & 46,9 & 49 & ,000 \\
\hline
\end{tabular}

VAVD: valva atrioventricular derecha; V: Ventral; D: Dorsal

Tabla II. Número de trabéculas que forman los M. papilares anterior, septal y posterior del ventrículo izquierdo en 50 corazones de avestruz (Struthio camelus).

\begin{tabular}{|c|c|c|c|c|c|c|c|c|}
\hline \multicolumn{3}{|c|}{ M. papilar anterior } & \multicolumn{3}{|c|}{ M. Papilar septal } & \multicolumn{3}{|c|}{ M. papilar posterior } \\
\hline $\begin{array}{l}\mathrm{N}^{\circ} \\
\text { trabéculas }\end{array}$ & Frecuencia & $\%$ & $\begin{array}{c}\mathrm{N}^{\mathrm{o}} \\
\text { trabéculas }\end{array}$ & Frecuencia & $\%$ & $\begin{array}{c}\mathrm{N}^{\mathrm{o}} \\
\text { trabéculas }\end{array}$ & Frecuencia & $\%$ \\
\hline 1 & 2 & 4,0 & - & - & - & - & - & - \\
\hline 2 & 39 & 78,0 & 2 & 8 & 16,0 & 2 & 17 & 34,0 \\
\hline 3 & 9 & 18,0 & 3 & 26 & 52,0 & 3 & 25 & 50,0 \\
\hline 4 & - & - & 4 & 14 & 28,0 & 4 & 7 & 14,0 \\
\hline 5 & - & - & 5 & 2 & 4,0 & 5 & 1 & 2,0 \\
\hline Total & 50 & 100,0 & - & 50 & 100,0 & - & 50 & 100,0 \\
\hline
\end{tabular}




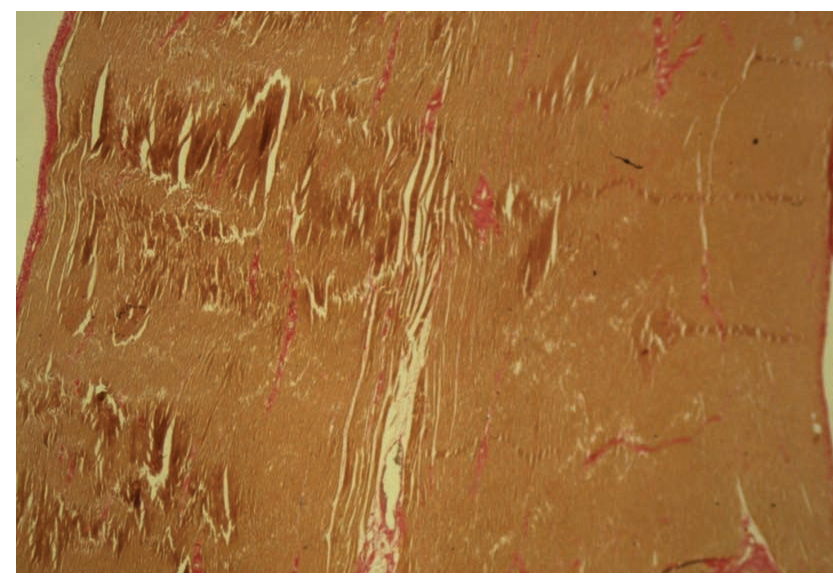

Fig. 9. Corte histológico del músculo papilar posterior del ventrículo izquierdo. Van Gieson. 12,5x.

\section{DISCUSIÓN}

El avestruz existente en Chile (Struthio camelus var. domesticus), introducido hace algunas décadas al país ha permitido diversificar a la industria avícola e influenciado la alimentación de un creciente número de personas, aportando como se ha dicho su carne y otros productos bajos en colesterol.

La valva atrioventricular derecha en el corazón del avestruz es muscular, semejante a una cinta o banda, que se encuentra unida, como ya la mencionó Macalister, a la parte pared ventral del ventrículo derecho. Sin embargo, la aseveración de este autor, nos parece poco precisa ya que en el avestruz (Struthio camelus var. domesticus), el ventrículo derecho presenta sólo dos paredes lateral y medial, donde se inserta la valva atrioventricular derecha.

Por otra parte, coincidimos con los autores consultados quienes señalaron que la inserción ventral de la valva atrioventricular derecha a la pared ventricular se encontraría en una posición mucho más craneal $(103,4 \mathrm{~mm})$ que la inserción dorsal $(63,5 \mathrm{~mm})$ en el corazón del avestruz (Struthio camelus var. domesticus).

Macalister estaría en lo correcto al describir que los Mm. papilares en el ventrículo derecho sólo son fibras musculares de la valva atrioventricular derecha, ya que en el ventrículo unicamente se observa esta banda muscular, sin la presencia de cuerdas tendinosas y músculos papilares independientes que estén uniendo la valva a la pared. Una excepción fue el hallazgo de una tercera inserción a la pared lateral del ventrículo derecho, similar a una trabécula septomarginal.

Se confirma con este estudio que en el ventrículo derecho del avestruz (Struthio camelus var. domesticus) no existen

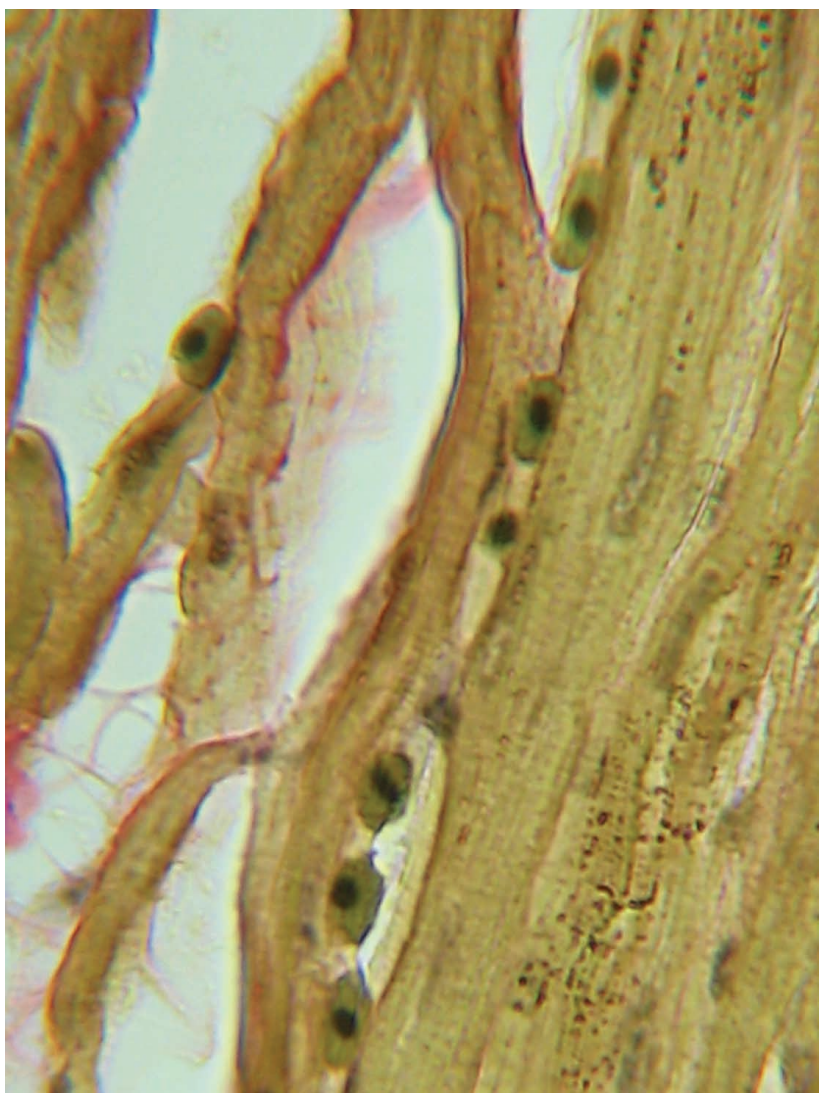

Fig. 10. Corte histológico del músculo papilar posterior del ventrículo izquierdo que muestra fibras de conducción cardíaca. (FP). Van Gieson. 400x.

músculos papilares y las trabéculas carnosas de este ventrículo no presentan fibras conductoras cardíacas, como ya ha sido señalado por Bezuidenhout y Sisson. Así, estas fibras penetrarían directamente desde la pared ventricular por las inserciones dorsal y ventral de la valva atrioventricular.

En el ventrículo izquierdo del avestruz (Struthio camelus var. domesticus), los diferentes autores consultados (Shaner; Adams; Bezuidenhout, 1981, 1983; Sisson; König y Tadjalli et al.) reconocen la existencia de tres músculos papilares los cuales sirven de inserción a las respectivas cuerdas tendinosas; a excepción de Macalister quien describió la presencia de cuerdas tendinosas, en las cuales no se observan músculos papilares conectados a ellas.

En el ventrículo izquierdo del avestruz (Struthio camelus var. domesticus) los tres músculos papilares se encuentran más desarrollados que en el corazón de las aves domésticas, probablemente, como consecuencia del tamaño corporal. Esto ha hecho que los músculos papilares se formen por la convergencia de largas trabéculas carnosas que desde el ápice se dirigen hacia la parte craneal del ventrículo izquierdo formando una masa muscular bastante desarrollada. 
En cuanto a la posición de los músculos papilares en el ventrículo izquierdo del avestruz (Struthio camelus var. domesticus), coincidimos con Shaner; Adams; Bezuidenhout, 1981, 1983; Baumel; Sisson; König y Tadjalli et al., quienes señalaron que dos de estos músculos se ubican en la pared lateral y el otro en la pared septal del ventrículo izquierdo, diferente a Lorenz (1921) quien ubica a los tres músculos en la pared lateral, hecho no observado en este estudio.

El trabajo muestra que en el ventrículo izquierdo del avestruz los tres músculos papilares se encuentran más o menos a la misma altura con relación al ápice cardiaco, siendo levemente más craneal el músculo papilar septal.

Con respecto al número de trabéculas carnosas que forman los músculos papilares (Tabla II), se puede observar que tanto el septal como el posterior están formados de tres trabéculas carnosas, en cambio, el músculo papilar anterior normalmente presenta dos trabéculas carnosas. Es importante señalar, como demuestran los resultados, que a lo largo de estas trabéculas carnosas se encuentran fibras conductoras cardiacas. No se encontró en la literatura trabajos relativos al número de trabéculas que forman los músculos papilares.

En resumen el corazón del avestruz (Struthio camelus var. domesticus) y en especial el sistema valvar atrioventricular de este animal, no difiere mayormente del resto de las aves domésticas, pero sí del corazón de la mayoría de los mamíferos donde las valvas atrioventriculares derecha e izquierda presentan, normalmente, tres y dos válvulas o cúspides con sus respectivas cuerdas tendinosas y músculos papilares, respectivamente.

FIGUEROA, M. \& HENRÍQUEZ-PINO, J. Papillary muscles in the heart of the ostrich (Struthio camelus). Int. J. Morphol., 27(2):435-440, 2009.

SUMMARY: The heart of the ostrich (Struthio camelus), just like the domestic birds's heart, has a muscular right atrioventricular valve without chordae tendinae and a membranous left atrioventricular valve with chordae tendinae than extends between the cusps and 3 papillary muscles. The aim of the study was to describe the papillary muscles, number, position and some morphologic characteristics. Fifty ostrich hearts ( Struthio camelus ), were dissected and fixed in $10 \%$ formalin and which got histological slices from 7 microns themselves they were stained with Van Gieson and $\mathrm{H}$ E. The macroscopic study evidenced absence of papillary muscles in the right ventricle and in the left ventricle 3 muscular masses formed by longitudinal trabeculae carneae that give insertion to the chordae tendinae. It was observed in the histological slices that the dorsal and ventral insertion points of the right atrioventricular valve to the ventricular wall, are prolongations of the muscle that forms the valve and shows conductive cardiac fibers. In the left ventricle, the longitudinal trabeculae carneae also show conductive cardiac fibers the ones that extend to the zone of insertion of the chordae tendinae. The results evidence that the heart of the ostrich (Struthio camelus ) does not have papillary muscles of conical form like in the human heart, are more developed muscular formations than in the domestic birds's heart and functionally acts like papillary muscles to show conductive cardiac fibers.

KEY WORDS: Anatomy; Heart; Ostrich; Papillary muscles.

\section{REFERENCIAS BIBLIOGRÁFICAS}

Adams, W. E. A contribution to the Anatomy of the Avian Heart as seen in the Kiwi (Apteryx australis) and the Yellow-crested Penguin (Megadyptes antipodum). Proc. Zool. Soc. Lond. Ser. B, 107:417-41, 1937.

Baumel, J. J.; King, A. S.; Breazile, J. E.; Evans H. E. \& Vanden Berge, J. C. Handbook of Avian Anatomy:Nomina anatomica avium. $2^{\mathrm{a}}$ ed. Publications of the Nuttal Ornithological Club. $\mathrm{N}^{\mathrm{o}}$ 23, Cambridge, Massachusetts, 1993.

Bezuidenhout, A. J. The anatomy of the heart of the ostrich Struthio camelus (Linn). DVSc. Thesis, Faculty of Veterinary Science, University of Pretoria, Pretoria, 1981.

Bezuidenhout, A. J. The Valva atrioventricularis dextra of the avian heart. Anat. Histol. Embryol., 12(2):104-8, 1983.

Brólio, M. P.; Ambrósio, C. E.; Miglino, M. A.; Carvalho, A. F.; Ferreira, G. J. B. C.; Martins, D. S. \& Assis Neto, A. C. Irrigação, topografia e morfologia do coração de Avestruzes (Struthio camelus). Int. J. Morphol., 21(1):78, 2003.

Fundación para la innovación agraria, Ministerio de Agricultura- Chile. Explotación comercial del avestruz-Antecedentes generales. Santiago, Chile, 1996.
König, Korbel, Liebich. Anatomie der Vögel. Stuttgart, Ed. Schattauer, 2009.

Lorenz, H. Beiträge zur Kenntnis des Vogelherzens. Med. Vet. Diss., Hannover, 1921.

Macalister, A. On the anatomy of the ostrich (Struthio camelus). Proc. R. Irish Acad., IX:1-24,1864.

Shaner, R. F. On the muscular architecture of the vertebrate ventricle. J. Anat., 58:59-70, 1923.

Sisson, S. \& Grossman, J. D. Anatomía de los animales domésticos.

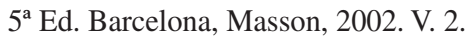

Tadjalli, M.; Ghazi, S. R. \& Parto, P. Gross anatomy of the heart in Ostrich (Struthio camelus). Iranian J. of Veterinary Research, Shiraz University, 10(1), Ser. No. 26: 21-7, 2009.

Dirección para correspondencia:

Dr. Marco Figueroa F.

Universidad del Bio-Bio

Av. Andrés Bello s/n

Chillán - CHILE

Email: mfiguer@ubiobio.cl

Recibido : $12-12-2008$

Aceptado: 14-02-2009 